\title{
Decrease in Mobility during the COVID-19 Pandemic and Its Association with Increase in Depression among Older Adults: A Longitudinal Remote Mobility Monitoring Using a Wearable Sensor
}

\author{
Ramkinker Mishra ${ }^{1}$ (D), Catherine Park ${ }^{1}$, Michele K. York ${ }^{2,3,4}{ }^{\mathbb{D}}$, Mark E. Kunik ${ }^{4,5,6}$, Shu-Fen Wung ${ }^{7}$, \\ Aanand D. Naik ${ }^{5,6,8}$ and Bijan Najafi ${ }^{1, *(D)}$
}

check for updates

Citation: Mishra, R.; Park, C.; York, M.K.; Kunik, M.E.; Wung, S.-F.; Naik, A.D.; Najafi, B. Decrease in Mobility during the COVID-19 Pandemic and Its Association with Increase in Depression among Older Adults: A Longitudinal Remote Mobility Monitoring Using a Wearable Sensor. Sensors 2021, 21, 3090. https:/ / doi.org/10.3390/s21093090

Academic Editor: Marco Iosa

Received: 6 March 2021

Accepted: 26 April 2021

Published: 29 April 2021

Publisher's Note: MDPI stays neutral with regard to jurisdictional claims in published maps and institutional affiliations.

Copyright: (C) 2021 by the authors. Licensee MDPI, Basel, Switzerland. This article is an open access article distributed under the terms and conditions of the Creative Commons Attribution (CC BY) license (https:// creativecommons.org/licenses/by/ $4.0 /)$.
1 Interdisciplinary Consortium on Advanced Motion Performance (iCAMP), Michael E. DeBakey Department of Surgery, Baylor College of Medicine, Houston, TX 77030, USA; ram.mishra@bcm.edu (R.M.); catherine.park@bcm.edu (C.P.)

2 Department of Neurology, Baylor College of Medicine, Houston, TX 77030, USA; myork@bcm.edu

3 Parkinson's Disease Research Education and Clinical Center, Michael E. DeBakey Veterans Affairs Medical Center, 2002 Holcombe Boulevard, Houston, TX 77030, USA

4 Department of Psychiatry and Behavioral Sciences, Baylor College of Medicine, Houston, TX 77030, USA; mark.kunik@bcm.edu

5 VA Health Services Research and Development Center for Innovations in Quality, Effectiveness and Safety, Houston, TX 77021, USA; anaik@bcm.edu

6 VA South Central Mental Illness Research, Education and Clinical Center (a Virtual Center), Houston, TX 77021, USA

7 College of Nursing, University of Arizona, Tucson, AZ 85721, USA; wung@arizona.edu

8 Department of Medicine, Baylor College of Medicine, Houston, TX 77030, USA

* Correspondence: najafi.bijan@gmail.com or Bijan.najafi@bcm.edu; Tel.: +1-713-798-7536

Abstract: Background: Social isolation during COVID-19 may negatively impact older adults' wellbeing. To assess its impact, we measured changes in physical activity and sleep among communitydwelling older adults, from pre-to post-pandemic declaration. Method: Physical activity and sleep in older adults $(n=10$, age $=77.3 \pm 1.9$ years, female $=40 \%$ ) were remotely assessed within 3-month pre-to 6-month post-pandemic declaration using a pendant-wearable system. Depression was assessed pre-and post-pandemic declaration using the Center for Epidemiologic Studies Depression scale and was compared with $48 \mathrm{~h}$ continuous physical activity monitoring data before and during pandemic. Results: Compared to pre-pandemic, post-pandemic time spent in standing declined by $32.7 \%$ (Cohen's $d=0.78, p<0.01)$, walking by $52.2 \%(\mathrm{~d}=1.1, p<0.01)$, step-counts by $55.1 \%$ $(\mathrm{d}=1.0, p=0.016)$, and postural transitions by $44.6 \%(\mathrm{~d}=0.82, p=0.017)$ with increase in sitting duration by $20.5 \%(d=0.5, p=0.049)$. Depression symptoms increased by $150 \%(d=0.8, p=0.046)$. Interestingly, increase in depression was significantly correlated with unbroken-prolong sitting bout ( $\rho=0.677, p=0.032)$, cadence $(\rho=-0.70, p=0.024)$, and sleep duration $(\rho=-0.72, p=0.019)$. Conclusion: This is one of the early longitudinal studies highlighting adverse effect of the pandemic on objectively assessed physical activity and sleep in older adults. Our observations showed need for timely intervention to mitigate hard to reverse consequences of decreased physical activity such as depression.

Keywords: wearable sensor; COVID-19; pandemic; mobility; depression; older adults; telemedicine; digital health; frailty; mental health

\section{Introduction}

The coronavirus disease 2019 (COVID-19) pandemic has exacted great illness, death, distress, and unprecedented restrictions on our society [1]. Older adults are at high risk for developing serious complications from COVID-19 and have been recommended to 
follow strict guidelines to minimize the risk of exposure during the current pandemic. The resulting social isolation can have a significant negative impact on the physical and mental health of older adults [2,3], the extent of which is not completely known at this time. Understanding these effects can help mitigate the negative effects of social isolation caused by the pandemic and prevent long-lasting negative impacts on older adults' health and wellbeing post-pandemic.

Previous studies suggest that, when older adults participate in social activities, it allows them to be physically active and preserve functional status [4,5]. However, as a preventive measure during the COVID-19 pandemic, community activities were halted, and social or family gatherings were discouraged. These measures may reduce physical activity level and sleep duration and, if they persist for a long time, could lead to hard to reverse consequences such as depression and frailty [6-8]. According to a report by the American Association of Retired Persons (AARP) and United Health Foundation, more than half of adults 50 years of age and older experienced social isolation during the pandemic, $41 \%$ felt more anxious, and $37 \%$ endorsed increased depressive symptoms [9]. A decline in mental health is often related to reduced physical activity, which increases the risk of mortality in older adults [3].

Pandemic-related restrictions can instigate a vicious cycle of reduced physical activity, abnormal sleep patterns, and decline in mental health in older adults [6], as shown in Figure 1. While systematic studies are lacking, Fitbit data scientists published the earliest report showing a $12 \%$ decline in the step count of millions of Fitbit users during the week of 22 March 2020, compared to the same week from the previous year [10]. Later, Browne et al. [11] demonstrated an $18 \%$ decline in daily steps count in 35 older adults with hypertension assessed before (January to March 2020) and during (June 2020) the COVID-19 pandemic. While Browne et al. demonstrated reduced physical activity in vulnerable older adults during the pandemic, they did not investigate the impact of COVID-19 on the sleep and mental health of older adults [11]. In one of the earliest surveys, AARP highlighted the impact of COVID-19 on the mental health of the general population and showed $41 \%$ of people were more anxious than usual, and $37 \%$ endorsed increased depressive symptoms [9]. Another survey conducted between 14 and 19 July 2020 by the Kaiser family foundation (KFF) showed that among, 1313 adults living in the United States, 36\% of participants faced difficulty sleeping [12]. Furthermore, on analyzing electronic health records, it was observed that among 62,354 COVID survivors (including people with and without a past psychiatric diagnosis), $18 \%$ were diagnosed with higher anxiety or mood disorders within 14 to 90 days following the diagnosis of COVID-19 [13]. Altogether, COVID-19 related complications raise serious concern about the safety of older adults, and deterioration of mental and physical health can increase adverse events like falls. For instance, SafelyYOU's community data indicate a $20 \%$ increase in falls for residents in memory care communities during the pandemic [14].

The above studies suggest that, during the pandemic, physical activity and sleep quality have deteriorated, probably because of voluntary (social isolation) and/or involuntary (lockdown) social distancing imposed by the pandemic. This deterioration may explain the higher prevalence of depression symptoms observed in older adults during the pandemic. However, the link between the increase in depression and changes in physical activity and sleep is still poorly understood. To address the gap, we leveraged an ongoing study initiated prior to the pandemic. More specifically, we recruited a cohort of communitydwelling older adults in which the patterns of physical activities and sleep were remotely monitored using a pendant sensor. When the pandemic was declared on 11 March 2020, we continued to follow-up with these participants up to 6 months post-pandemic, allowing us to objectively determine the pandemic's effect on physical activities and sleep pattern. We analyzed the collected data to address two specific aims. In the first aim, we examined changes from pre- to post-pandemic in mobility performance, including commutative postures (sitting, standing, lying, and walking); walking characteristics (e.g., daily step count); motor performance (e.g., number of daily postural transition); and sleep (e.g., total 
sleep duration during night time). In the second aim, we explored the association between changes in mobility performance and depression during the same time interval. We hypothesized that there would be a decline in the participants' physical activity and sleep with increased depression symptoms after the pandemic compared to pre-pandemic. Our second hypothesis was that increase in depression would be associated with reduced physical activity and sleep.

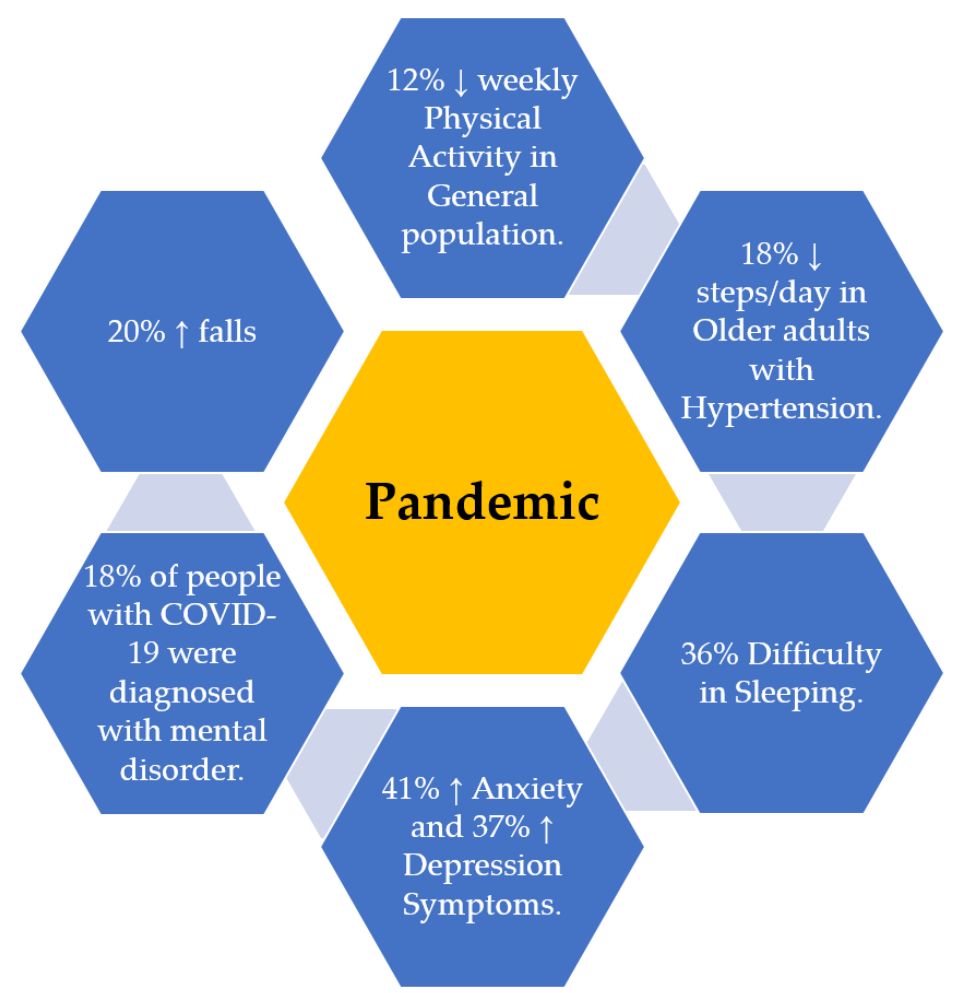

Figure 1. Summarized representation of studies which investigated the impact of the COVID-19 pandemic on physical activity, sleep, and mental health. These studies highlighted that, during the COVID-19 pandemic, the activity level has been reduced in both the general population [10] and those with chronic illness (e.g., hypertension) [11], and sleep quality has deteriorated [12]. Other studies suggested that during the pandemic, depression, anxiety, and mental health disorder have been increased compared to pre-pandemic $[9,13,14]$. However, these studies were limited to create a link between changes in the pattern of physical activities and sleep with changes in depression symptoms in the context of a prospective study design.

\section{Materials and Methods}

\subsection{Study Population}

Participants were recruited from an ongoing study focused on automatic fall detection and monitoring the risk of falling in community older adults with a high risk of falling using a wearable pendant sensor (ActivePERS/PAMSys, Biosensics LLC, Newton, MA, USA). Inclusion criteria were community older adults aged 75 years or older or aged 65 years older with a high risk of falling. The risk of falling was determined by either selfreported history of fall over last 12 months or self-report high concerns for fall. Participants were excluded if they were living in a nursing home, in hospice care, or if they were unable to independently walk a distance of $10 \mathrm{~m}$ with or without an assistive device or unable to stand still without moving feet, which may affect their daily physical activity, or were unwilling to participate. All participants were followed up for 12 months, including three time-point assessments of mobility, cognition, and mental health (e.g., depression) at baseline, 6 months, and 12 months. For this specific study, we excluded those without valid physical activity data or depression assessment no longer than three months before the pandemic declaration (11 March 2020) and with minimum three months to up to nine 
months post-pandemic declaration. The study was conducted according to the guidelines of the Declaration of Helsinki and approved by the Institutional Review Board (or Ethics Committee) of Baylor College of Medicine (protocol code H41717 and 13 December 2017).

\subsection{Demographics and Clinical Data}

Demographics and relevant clinical information, including age, gender, height, weight, ethnicity, fall history, and pre-existing medical conditions, were collected at baseline using chart-review and self-report. Body mass index (BMI) was calculated based on each older adult's height and weight. At each study visit, participants underwent clinical assessments, including Montreal Cognitive Assessment (MoCA) [15], Center for Epidemiologic Studies Depression scale (CES-D) [16], Fear of falling (FES-I) [17], Lawton Instrumental Activities of Daily Living Scale (IADL) [18], and Beck Anxiety Inventory (BAI) [19]. The CES-D short-version scale was used to measure self-reported depression symptoms before and during the pandemic. A cutoff CES-D score of 16 or greater was used to identify subjects with depression [16]. Furthermore, scores of 36 and above on BAI indicated potentially concerning levels of anxiety in the participants [19]. Cognitive impairment was defined as a MoCA score less than 25, recommended by Nasreddine et al. (2005). Moreover, participants' scores in the range of 28-64 on FES-I were considered to have a high concern about falling [20]. A person's ability to perform tasks was determined based on a summary score on the IADL survey in the ranges from 0 (low function, dependent) to 8 (high function, independent) for women and 0 through 5 for men [18].

\subsection{Sensor-Derived Monitoring of Physical Activity and Sleep}

To collect fine grain-information about physical activity, we monitored physical activities and sleep pattern for a continuous period of $48 \mathrm{~h}$, at each study visit, using a validated pendant sensor (PAMSys ${ }^{\mathrm{TM}}$, BioSensics LLC, Watertown, MA, USA), worn around the neck (Figure 2). Physical activity and sleep in older adults were remotely assessed within the 3-month pre-to 6-month post-pandemic declaration during regular life. The participants were instructed to continuously wear the pendant for two consecutive days (48 h). Later, the pendant was returned to the research center through either a paid mail service or retrieved by a research coordinator. Prior studies have shown that two-day monitoring of daily life activities is sufficient to assess frailty [21] and yields optimal adherence to wearing the sensor continuously [22]. The PAMSys ${ }^{\mathrm{TM}}$ pendant consists of a 3-axis accelerometer, battery, processor, and built-in memory for recording long-term data. Accelerometer signals were recorded at a sampling frequency of $50 \mathrm{~Hz}$. All physical activity and sleep-related parameters were extracted from the pendant using two different validated algorithms; an algorithm to extract spontaneous daily physical activities (e.g., cumulative postures, postural transitions, and walking characteristics) and an algorithm to quantify sleep quality (e.g., sleep/awake duration during night-time). These algorithms were described in detail in our previous studies [23-26].

The estimated physical activities included for final data analysis were characterized by (1) cumulated percentage of sitting, standing, lying, and walking postures; (2) daily walking characteristics (step count, cadence), and the number of the unbroken walking bouts, which included a minimum three consecutive steps within a 5-s interval [27]); (3) number of postural-transitions summing up postural-transitions such as sit-to-stand, stand-to-sit, walk-to-stand, stand-to-walk, walk-to-sit. When the duration of standing between a walk to sit and vice versa was less than $1 \mathrm{~s}$, it was considered as walk-to-sit or sit-to-walk transition [28]; and (4) activity behavior, including unbroken prolonged sitting bout, light activity, and moderate-to-vigorous activity [21,25]. The unbroken prolonged sitting bout was defined as the 90th percentile of all recorded sitting bouts duration [21,25]. Light activity was defined as an activity between metabolic equivalent (MET) $\geq 1.5$ and $<3.0$, such as ironing, washing, and dusting, and working at a standing workstation [19]. Moderate-to-vigorous activity referred to an activity demanding $\geq 3.0 \mathrm{MET}$, such as brisk walking, recreational activities, taking stairs, etc. [21]. 


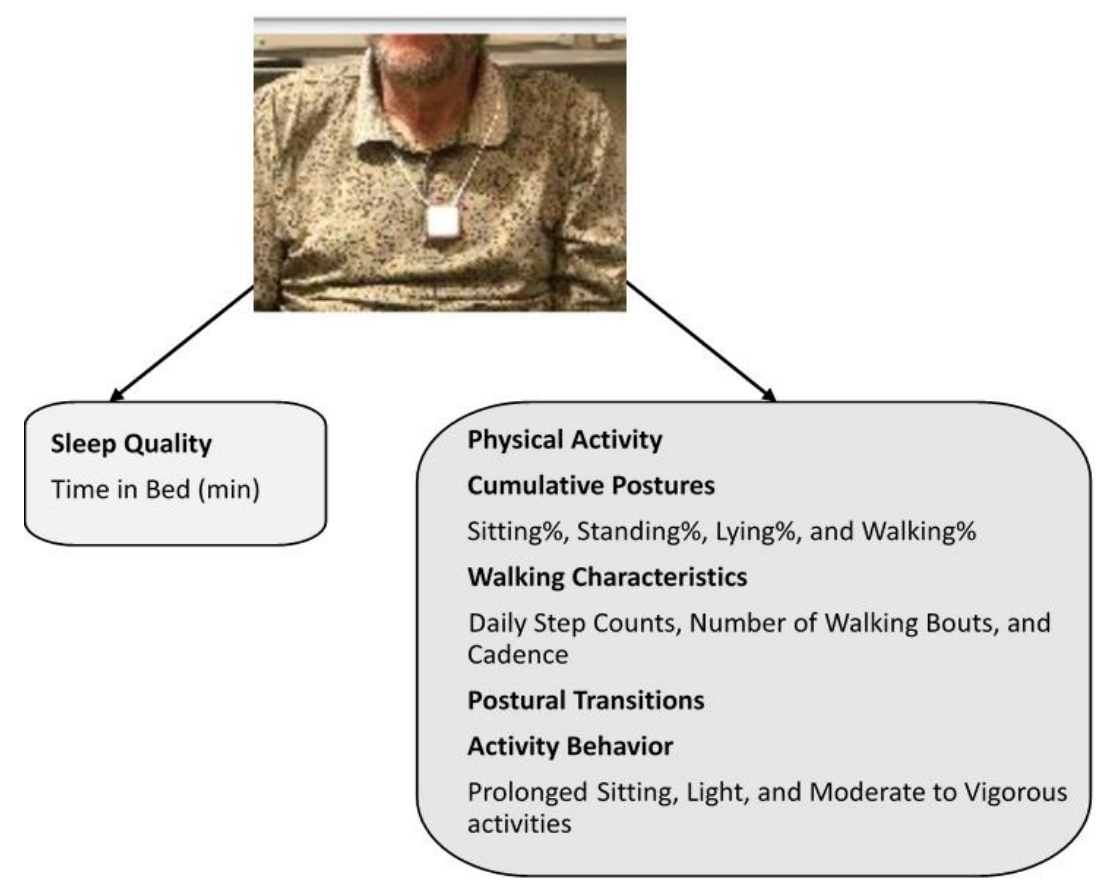

Figure 2. Participants wore the pendant sensor around the neck as shown above and the physical activity and sleep parameters that were extracted based on the validated algorithm are shown below, namely: time in bed, cumulative postures, walking characteristics, postural transitions, and activity behavior.

Furthermore, sleep was characterized in terms of the total duration of a participant's time in bed during the night [21]. We described the details for extracting time in bed during the night in our previous study [26]. Briefly, first, the unwanted noise was removed from the acceleration signal by applying a band-pass filter. Then, for every minute, a vector magnitude/norm of acceleration was estimated. Finally, a model was used to estimate the sleep/wake conditions based on the moment and standard deviation calculated from every one-minute acceleration vector, posture (sleeping on sides or back), and postural transition (e.g., tossing on bed, rotating from back to sides, etc.) information.

\subsection{Statistical Analysis}

All continuous data were presented as the mean \pm standard error, and categorical data were expressed as the percentage. A paired $t$-test was used for with-group comparison of continuous demographics, clinical data, and physical activity metrics. Before applying the paired $t$-test, the assumption of normality was assessed using Shapiro-Wilk's test of normality $(p>0.05)$. The Wilcoxon signed-rank test was performed if the normality assumption was not satisfied. The effect size for discriminating between groups was estimated using Cohen's d effect size and represented as d [29]. The Spearman correlation coefficient was used to evaluate the degree of association between physical activity metrics and depression level. The correlation coefficient was also interpreted as effect size [30]. All statistical analyses were performed using IBM SPSS Statistics 25 (IBM, Chicago, IL, USA), with a significance level defined as $p<0.05$.

\section{Results}

\subsection{Demographic and Clinical Characteristics}

Ten older adults (age $=77.3 \pm 1.9$ years, females $=40 \%$, and BMI $=27.5 \pm 1.6 \mathrm{~kg} / \mathrm{m}^{2}$ ) satisfied the inclusion and exclusion criteria of this study. On average, the baseline data were collected $1.13 \pm 0.43$ months before the pandemic, and follow-up assessment performed on average $5.9 \pm 0.67$ months post-pandemic declaration. Table 1 summarizes the demographics and clinical information of these participants. At baseline, participants reported low levels of depression $(2.8 \pm 0.7)$ and anxiety $(2.1 \pm 1.0)$ symptoms on CES-D and 
BAI scales, respectively. According to the MoCA assessment, three participants (30\%) were classified as cognitively impaired. Furthermore, two participants (20\%) reported having a low functional ability on IADL. While three participants (30\%) reported falls in the last 12 months, FES-I scores indicated no participant had a high concern of falling. As presented in Table 2, post-pandemic declaration, there was an increase in depression symptoms by $150 \%$ (Cohen's $d=0.8, p=0.046$ ), but no significant change in other psychosocial metrics (e.g., anxiety, fear of falling, and activities of daily life).

Table 1. Demographic and clinical information of the participants. Values are presented as mean \pm standard error (SE), or percentage (\%).

\begin{tabular}{cc}
\hline Demographics & \\
Age, years & $77.3 \pm 1.9$ \\
Sex (Female), $\%$ & $40 \%$ \\
Height, $\mathrm{m}$ & $1.63 \pm 0.09$ \\
Weight, $\mathrm{kg}$ & $83.4 \pm 21.5$ \\
Body Mass Index (BMI), kg/m² & $27.5 \pm 1.6$ \\
Clinical data & \\
Had fall in last 12-month, \% & $30 \%$ \\
Cancer (\%) & $40 \%$ \\
Number of prescription medications, $\mathrm{n}$ & $2.1 \pm 0.6$ \\
Cognition (MoCA), score & $25.1 \pm 1.6$ \\
Cognitive impairment, $\%$ & $30 \%$ \\
Center for Epidemiologic Studies Depression (CES-D), score & $2.8 \pm 0.7$ \\
Dear of Falling (FES-I), score & $0 \%$ \\
(High Concern) Fallers, $\%$ & $19.3 \pm 1.0$ \\
Low Functional Ability, $\%$ & $0 \%$ \\
Anxiety (BAI), score & $6.6 \pm 0.9$ \\
High Anxiety, $\%$ & $20 \%$ \\
Time-point of assessments & $2.1 \pm 1.0$ \\
Activities of Daily Living Scale (IADL), score & $0 \%$ \\
Average duration pre-pandemic assessment, months & $1.13 \pm 0.43$ \\
Average duration post-pandemic assessment, months & $5.9 \pm 0.67$ \\
\hline
\end{tabular}

Table 2. Psycho-social, Cumulated Posture, Walking characteristics, Postural transitions, Activity behavior, and Sleep related measures assessed before and during pandemic.

\begin{tabular}{|c|c|c|c|c|c|}
\hline & Before & During & Mean Difference \% & Cohen's d & $p$-Value \\
\hline \multicolumn{6}{|c|}{ Psycho-social Behavior } \\
\hline Depression, score & $3.0 \pm 0.7$ & $7.5 \pm 2.4$ & $150.0 \%$ & 0.80 & $0.046 *$ \\
\hline Fear of Falling, score & $19.7 \pm 1.2$ & $18.7 \pm 1.0$ & $-5.1 \%$ & 0.29 & 0.443 \\
\hline Anxiety, score & $2.1 \pm 1.0$ & $2.9 \pm 1.4$ & $38.1 \%$ & 0.21 & 0.588 \\
\hline Activity of daily life, score & $6.6 \pm 0.9$ & $6.0 \pm 1.0$ & $-9.1 \%$ & 0.19 & 0.131 \\
\hline \multicolumn{6}{|c|}{ Cumulated Posture } \\
\hline Sitting percentage, $\%$ & $37.5 \pm 4.5$ & $45.2 \pm 5.1$ & $20.5 \%$ & 0.5 & 0.049 * \\
\hline Lying percentage, \% & $39.3 \pm 4.6$ & $40.5 \pm 4.7$ & $3.1 \%$ & 0.24 & 0.768 \\
\hline Standing percentage, $\%$ & $16.5 \pm 2.3$ & $11.1 \pm 1.8$ & $-32.7 \%$ & 0.78 & $<0.01 *$ \\
\hline Walking percentage, $\%$ & $6.7 \pm 1.3$ & $3.2 \pm 0.5$ & $-52.2 \%$ & 1.1 & $<0.01$ * \\
\hline \multicolumn{6}{|c|}{ Walking Characteristics } \\
\hline Daily Step count, $\mathrm{n}$ & $5911 \pm 1193^{\circ}$ & $2655 \pm 419$ & $-55.1 \%$ & 1.0 & $0.016 *$ \\
\hline Number of unbroken walking bout, $\mathrm{n}$ & $241.3 \pm 56.2$ & $148.6 \pm 27.1$ & $-38.4 \%$ & 0.52 & $0.046 *$ \\
\hline Cadence, steps $/ \mathrm{min}$ & $81.1 \pm 2.3$ & $83.9 \pm 1.7$ & $3.4 \%$ & 0.4 & 0.367 \\
\hline \multicolumn{6}{|c|}{ Postural Transition } \\
\hline Number of Postural Transitions, $\mathrm{n}$ & $720.7 \pm 162.2$ & $399.4 \pm 68.5$ & $-44.6 \%$ & 0.82 & $0.017 *$ \\
\hline Average duration of stand-to-sit transition, s & $3.0 \pm 0.07$ & $2.7 \pm 0.3$ & $-10.0 \%$ & 0.4 & 0.88 \\
\hline Average duration of sit-to-stand transition, $\mathrm{s}$ & $3.0 \pm 0.08$ & $3.0 \pm 0.07$ & $0 \%$ & 0 & 0.57 \\
\hline \multicolumn{6}{|c|}{ Activity Behavior } \\
\hline Prolong Sitting, s & $240.9 \pm 46.5$ & $287.3 \pm 61.6$ & $19.3 \%$ & 0.26 & 0.392 \\
\hline Average Light Activity, min & $10.8 \pm 0.7$ & $10.5 \pm 0.8$ & $-2.8 \%$ & 0.13 & 0.678 \\
\hline Average Moderate to Vigorous Activity, min & $31.0 \pm 5.4$ & $27.3 \pm 4.6$ & $-11.9 \%$ & 0.23 & 0.526 \\
\hline \multicolumn{6}{|c|}{ Sleep Quantity } \\
\hline Time in Bed, s & $566.3 \pm 66.2$ & $583.7 \pm 67.5$ & $3 \%$ & 0.08 & 0.768 \\
\hline
\end{tabular}

* represents the significant difference $(p<0.05)$. 


\subsection{Physical Activity and Sleep Characteristics}

There was an increase in daily sitting duration by $20.5 \%(\mathrm{~d}=0.5, p=0.049)$. Furthermore, there was a decline in daily standing duration on average by $32.7 \%(\mathrm{~d}=0.78$, $p<0.01)$, walking duration by $52.2 \%(\mathrm{~d}=1.1, p<0.01)$, daily step-counts by $55.1 \%(\mathrm{~d}=1.0$, $p=0.016)$, and number of daily postural transitions by $44.6 \%(\mathrm{~d}=0.82, p=0.017)$ (Table 2 , Figures 3 and 4). There was no significant change in other sensor-derived parameters.

(A)

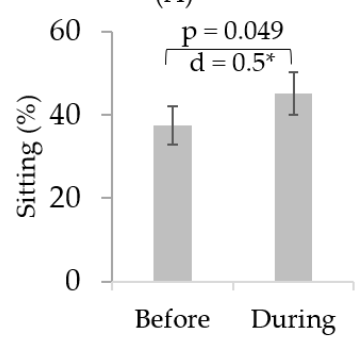

(C)

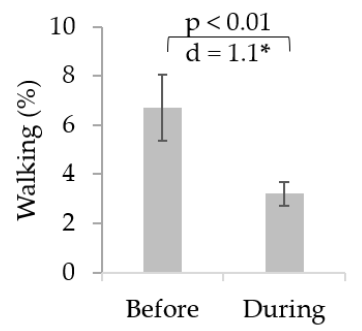

(B)

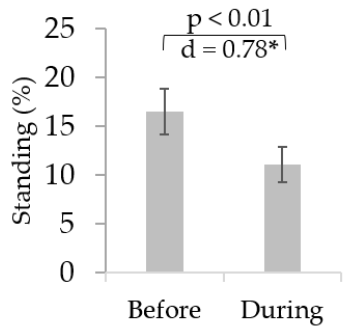

(D)

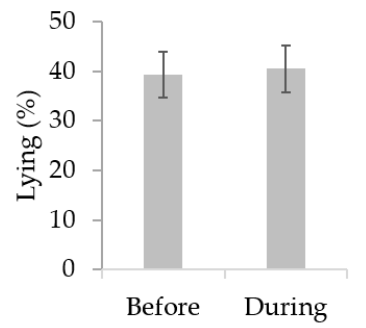

Figure 3. Representation of percentage of cumulated time spent in postures involving (A) sitting; (B) standing; (C) walking; and (D) Lying by the participants as remotely assessed by the PAMSys sensor before and during the pandemic. While there was no significant change in overall time spent lying, there was a significant increase in sedentary behavior and a decline in time spent walking or standing. * represents significant difference $(p<0.05)$.

(A)

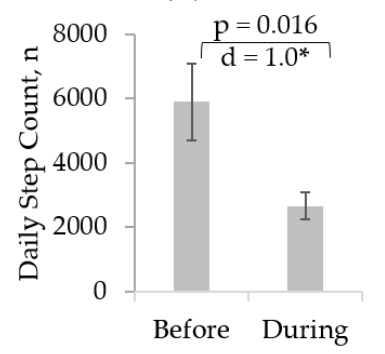

(D)

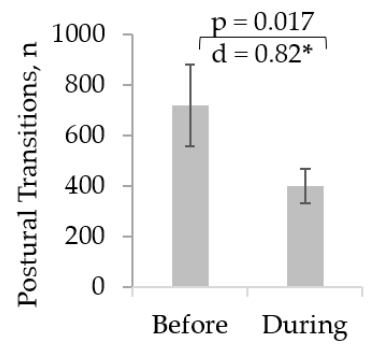

(B)

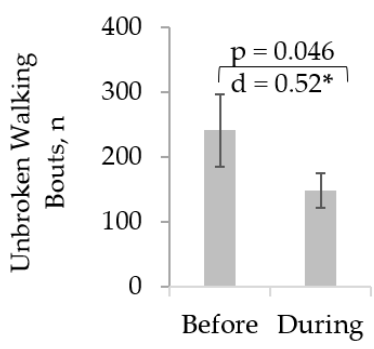

(E)

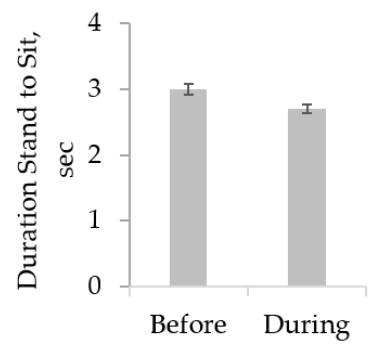

(C)

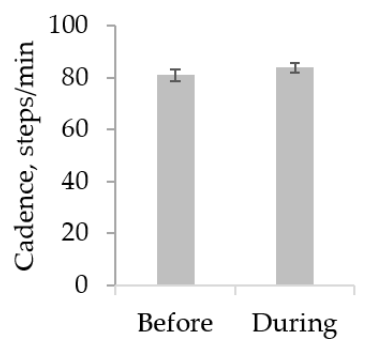

(F)

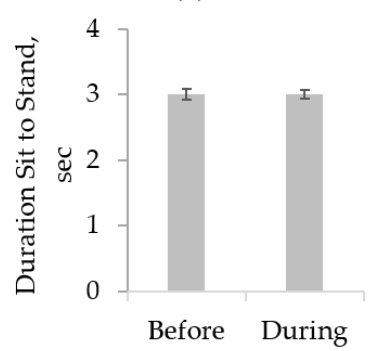

Figure 4. Walking characteristics and postural transitions of the participants as remotely assessed by the PamSys sensor before and during pandemic. We observed a significant decrease in (A) daily step count, (B) unbroken walking bout, and (D) postural transitions. There was no significant change on (C) cadence, (E) duration of stand to sit transition, and (F) duration of sit to stand transition. Error bars indicate standard errors of the corresponding averages $\left({ }^{*}\right.$ represents significant differences $(p<0.0 .05))$. 


\subsection{Association between Change in Depression and Sensor-Derived Parameters}

Table 3 summarizes the correlations between change in depression score with change in cadence, unbroken prolonged sitting bout, activity behavior, and sleep. As represented in Figure 5, we observed that as depression symptoms increased, there was a decline in cadence $(\rho=-0.701, p=0.024)$, time in bed $(\rho=-0.72, p=0.019)$ with a trend towards reduced light activity $(\rho=-0.566, p=0.088)$, and moderate-to-vigorous activity $(\rho=-0.41$, $p=0.24$ ) levels. Furthermore, increase in depression score was associated with increase in prolonged sitting $(\rho=0.68, p=0.032)$.

Table 3. Association with the change in depression measured using Spearman correlation analysis.

\begin{tabular}{cccc}
\hline & Correlations Coefficient & Variance, $\mathbf{R}^{2}$ & $p$-Value \\
\hline$\Delta^{1}$ Cadence, steps $/$ min & $-0.701^{*}$ & 0.49 & 0.024 \\
$\Delta$ Prolonged Sitting, s & $0.677^{*}$ & 0.46 & 0.032 \\
$\Delta$ Average Light Activity, min & -0.566 & 0.32 & 0.088 \\
$\Delta$ Average Moderate to Vigorous Activity, min & -0.409 & 0.16 & 0.241 \\
$\Delta$ Time in Bed, s & $-0.720 *$ & 0.52 & 0.019 \\
\hline
\end{tabular}

${ }^{1} \Delta$ represents change in metric (During-Before pandemic). * represents the significant $(p<0.05)$ correlation with the $\Delta$ Depression.

(A)

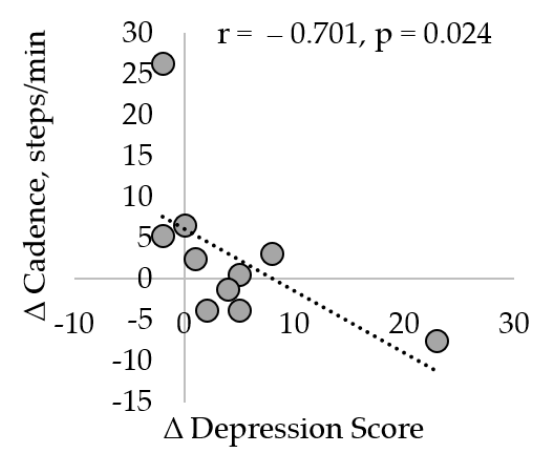

(B)

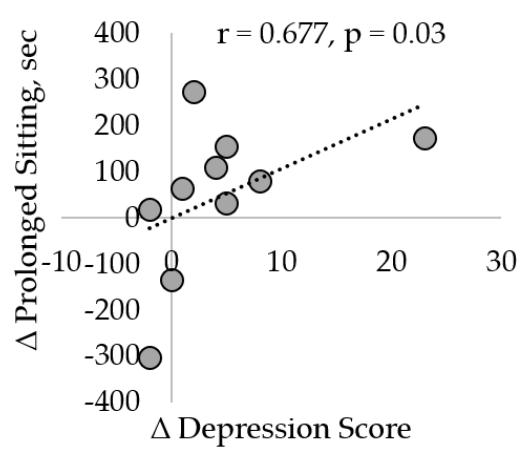

(C)

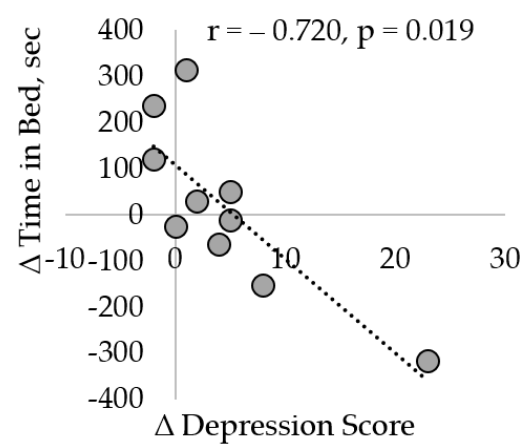

Figure 5. Association between change in depression and sensor derived parameters. Increase in depression symptoms was significantly correlated with (A) change in cadence, (B) change in prolonged sitting, and (C) change in time in bed. The symbol $\Delta$ represents the change in metric (During-Before pandemic).

\section{Discussion}

This study explored longitudinal objective changes in patterns of physical activities and sleep from pre- to post-pandemic in community-dwelling older adults and associate them with the increase in depression symptoms as summarized in Figure 6. The major findings of the present study were a significant decline in physical activity pattern including cumulative postures (sitting, standing, and walking), walking quantity (daily step count), high-level motor function (daily number of postural transitions), and sleep quantity (time in bed during night). Furthermore, it revealed a noticeable pairwise increase in depression score on average by $150 \%$ within 6 months post-pandemic compared to 3 months prepandemic. Most importantly, this study found a significant association between the increase in depression symptoms post-pandemic with deterioration in physical activity and sleep. For instance, the results suggest that $52 \%, 49 \%, 46 \%$, and $32 \%$ of the variation in depression score from pre- to post-pandemic could be explained by reduced time in bed at night, a decline in cadence, increase in the unbroken prolonged sitting bout, and reduction in the percentage of light activities from pre- to post-pandemic declaration. These findings are aligned with the conclusions of Sepúlveda-Loyola et al. (2020), who suggest reduced social interaction due to COVID-19 related restrictions increases depression and reduces physical activity level in older adults [5]. These results confirm the expected unhealthy physical activity level changes and decline in older adults' mental health during the COVID-19 pandemic. 

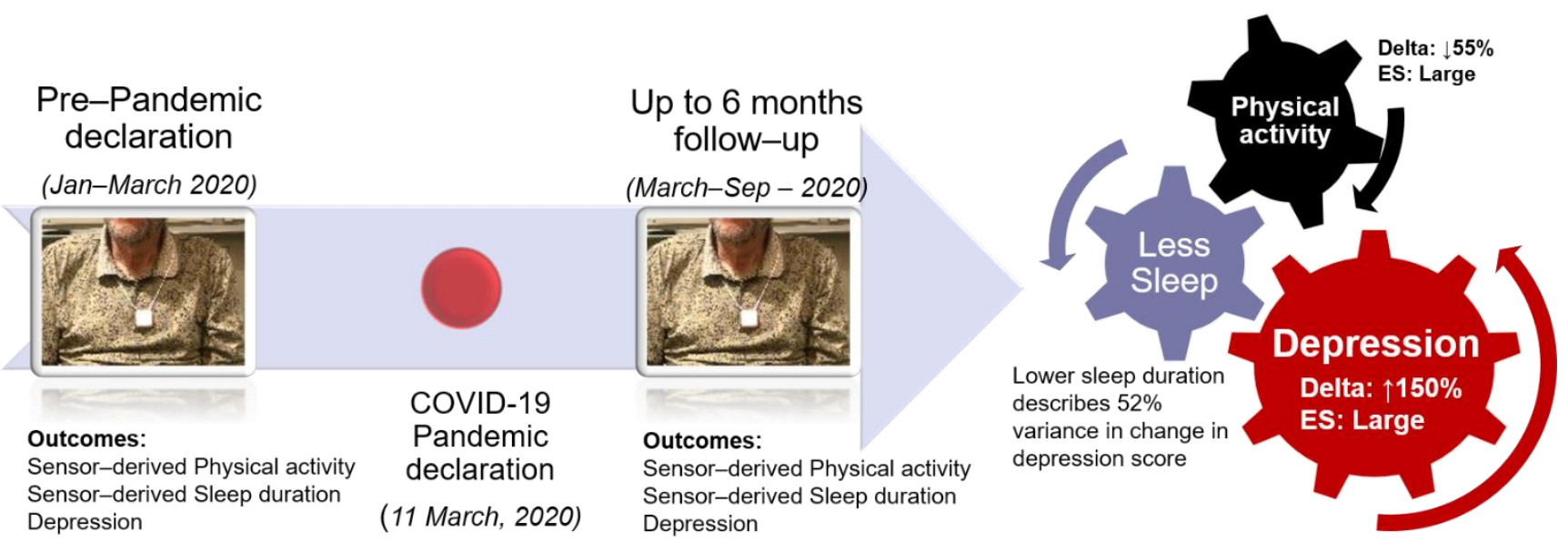

Figure 6. In this study, we followed a cohort of community-dwelling older adults $(\mathrm{n}=10)$ from the pre-pandemic to up to 6 months post declaration of the COVID-19 pandemic (11 March 2020). The results suggest that, compared to pre-pandemic physical activity (daily step count) it is reduced by $55 \%$, and depression is increased on average by $150 \%$. Our results also suggest that lower sleep duration (time in bed during the night-time) describes $52 \%$ of the variance in change in depression score. Abbreviation: ES represents effect size.

Several studies based on the surveys, interviews, and questionnaires support the negative impact of COVID-19 on physical activity patterns, sleep, and psycho-social behavior in older adults $[5,6,9,11]$. To our knowledge, this is the first prospective study that reports objective sensor-derived data related to physical activities and sleep patterns from the same individual followed from the pre-declaration of the pandemic to several months post COVID-19 pandemic declaration. This study design enables untangling of the association between the changes in physical activity and sleep patterns to changes in depression because of the COVID-19 pandemic in older adults. Our study suggests that increased depression during the COVID-19 pandemic in older adults can be explained by increased prolonged unbroken sitting bout, reduction in cadence during walking, and reduction in sleep duration during the night. Our results also suggest that during the COVID-19 pandemic, the daily step count was reduced on average by $55.1 \%$ during the pandemic compared to pre-pandemic, probably because of voluntary or involuntary social restriction imposed by the pandemic. During the same time, the depression symptom was increased by $150 \%$. Together, we speculate that the increase in depression was partially because of a reduction in the level of physical activities. This speculation is supported by few pre-pandemic prospective studies in which it was demonstrated that a decline in the level of physical activities might lead to an increase in depression symptoms. For instance, Edwards and Loprinzi [31] recruited 26 young adults (age: $21.7 \pm 2.71$ years, $38 \%$ male). The participants were asked to keep their walking steps count to less than 5000 steps per day for a one-week duration. The results suggest that this restriction led to an increase in depression symptoms on average by $83.7 \%(p<0.01)$. In another prospective study, Endrighi et al. [32] recruited 43 younger adults between 18 and 35. During the first two weeks, participants were asked to be as sedentary as much as possible. Then, for the follow-up two weeks, they were asked to return to their daily routine. Using ActiGraph, they showed that sedentary time was on an average 5.5\% higher during the first two weeks compared to the habitual activities (during the next two weeks). The results suggest that this increase in sedentary time led to a $55.1 \%$ increase in psychological distress and mood disturbance [32].

Even though a progressive return to everyday life is taking place gradually, all the preventive and safety measures for the ongoing pandemic are instilling feelings of uncertainty, fears, and concerns. Furthermore, limited available resources for older adults to mitigate mental health concerns pose a more significant challenge in the current circumstances. As implied by our results, increased sedentary behavior in older adults should be a target for treatment management during the COVID-19 pandemic. Prolonged reduction in physical 
activity and sleep could lead to hard to reverse complications such as depression, cognitive decline, and frailty $[3,19]$.

Our previous study showed that people with an increased level of frailty (non-frail, prefrail to frail state) exhibit a reduced number of postural transitions in daily life activities [26]. In the context of COVID-related changes, the postural transition can be considered a more reliable measurement of functional performance in older adults less influenced by environmental conditions compared to daily step counts or walking duration [33].

This study has limitations that need to be acknowledged. First, although we observed pairwise changes in depression level and physical activity level with medium to large effect sizes, the sample size was small; therefore, our results should be interpreted with caution. Second, this is a secondary analysis of an ongoing study, which was designed to remotely monitor the physical activity and fall incidents with a timeline of 12 months; thus, it may be underpowered to validate this study's hypotheses. Participants screened for this study, with their assessment time points overlapping with before and during the pandemic, were used in this exploratory analysis. Therefore, the duration between pre-and post-pandemic assessments varied from 4 to 12 months (Table 1). Another limitation is that, for given study design, the directionality between change in depression and physical activity, i.e., it may be that depression is causing decline in physical activity. Despite the mentioned limitations above, our preliminary findings might contribute to a better understanding of the unhealthy physical and mental health changes during the COVID-19 pandemic and guide future feasible preventive and therapeutic actions for older adults. In the future, a systematic study would be needed that can investigate changes in physical and mental health in older adults during and after the pandemic.

Despite the above limitations, this study may better shed light on the effect of the COVID-19 pandemic on physical activity, sleep, and depression thanks to its longitudinal study design. Another key advantage of our study is the use of objective sensor-derived metrics design enabling us to objectively track for the same individual the changes in physical activities and sleep patterns from the pre-pandemic to few months post the declaration of pandemic and link it to changes in depression symptoms.

\section{Conclusions}

This prospective study highlighting the adverse effect of the COVID-19 pandemic on physical activity and sleep, objectively monitoring using a pedant sensor in community dwelling older adults. We observed a 150\% increase in depression, and this increase is correlated with the prolonged sitting bout, nighttime sleep duration, and cadence. Additionally, our results suggest that reduced sleep time explains the $52 \%$ variance in change in depression. Timely intervention strategies are urgent to support recovery in physical activity and functional performance to the pre-pandemic level to mitigate the consequences of social isolation, including the increase in depression. The critical limitation includes the small sample size and timeline of assessment. In the future, a systematic study would be needed that can investigate changes in physical and mental health in older adults during and after the pandemic.

Author Contributions: R.M. contributed to conceptualization, data curation, formal analysis, investigation, methodology, project administration, resources, software, supervision, validation, visualisation, writing—original draft, and writing—review and editing. C.P., M.K.Y., M.E.K., S.-F.W., B.N. and A.D.N. contributed to conceptualization, visualization, writing-original draft, and writing-review and editing. B.N. further contributed to funding acquisition, project administration, resources, supervision, and validation. R.M. and B.N. have accessed and verified the underlying data. All authors have read and agreed to the published version of the manuscript.

Funding: Partial support was provided by the National Institute on Aging (award numbers NIA 2SB1AG032748 and 1R42AG060853-01) and internal support from Baylor College of Medicine. The content is solely the responsibility of the authors and does not necessarily represent the official views of sponsors. 
Institutional Review Board Statement: The study was conducted according to the guidelines of the Declaration of Helsinki, and approved by the Institutional Review Board (or Ethics Committee) of Baylor College of Medicine (protocol code H41717 and 13 December 2017).

Informed Consent Statement: Informed consent was obtained from all subjects involved in the study.

Data Availability Statement: The data presented in this study are available on request from the corresponding author. The data are not publicly available due to propriety reasons and need for IRB permission before sharing.

Acknowledgments: The authors would like to acknowledge study coordinator Anmol Momin and Ilse Paulette for their invaluable support in data collection.

Conflicts of Interest: The authors declare no conflict of interest.

\section{References}

1. Berg-Weger, M.; Morley, J.E. Loneliness and Social Isolation in Older Adults during the COVID-19 Pandemic: Implications for Gerontological Social Work. J. Nutr. Health Aging 2020, 456-458. [CrossRef] [PubMed]

2. Holt-Lunstad, J.; Smith, T.B. Loneliness and social isolation as risk factors for CVD: Implications for evidence-based patient care and scientific inquiry. Heart 2016, 987-989. [CrossRef] [PubMed]

3. Holt-Lunstad, J.; Smith, T.B.; Baker, M.; Harris, T.; Stephenson, D. Loneliness and Social Isolation as Risk Factors for Mortality. Perspect. Psychol. Sci. 2015, 10, 227-237. [CrossRef]

4. Tomioka, K.; Kurumatani, N.; Hosoi, H. Association Between Social Participation and 3-Year Change in Instrumental Activities of Daily Living in Community-Dwelling Elderly Adults. J. Am. Geriatr. Soc. 2017, 65, 107-113. [CrossRef] [PubMed]

5. Sepúlveda-Loyola, W.; Rodríguez-Sánchez, I.; Pérez-Rodríguez, P.; Ganz, F.; Torralba, R.; Oliveira, D.V.; Rodríguez-Mañas, L. Impact of social isolation due to COVID-19 on health in older people: Mental and physical effects and recommendations. J. Nutr. Health Aging 2020, 1-10. [CrossRef]

6. Antunes, R.; Frontini, R. Physical activity and mental health in Covid-19 times: An editorial. Sleep Med. 2021, 77, 295-296. [CrossRef]

7. Morin, C.M.; Carrier, J. The acute effects of the COVID-19 pandemic on insomnia and psychological symptoms. Sleep Med. 2021 [CrossRef]

8. Wang, C.; Patriquin, M.; Vaziri, A.; Najafi, B. Mobility Performance in Community-Dwelling Older Adults: Potential Digital Biomarkers of Concern about Falling. Gerontology 2021, 1-9. [CrossRef]

9. Report: Pandemic Has Created Loneliness Epidemic, New Report Shows. 2021. Available online: https://www.aarp.org/homefamily / friends-family/info-2020/isolation-survey-coronavirus / (accessed on 8 October 2020).

10. The Impact of Coronavirus on Global Activity—Fitbit Blog (23 March 2020). Available online: https://blog.fitbit.com/covid-19 -global-activity/ (accessed on 21 January 2021).

11. Browne, R.A.; Macêdo, G.A.; Cabral, L.L.; Oliveira, G.T.; Vivas, A.; Fontes, E.B.; Elsangedy, H.M.; Costa, E.C. Initial impact of the COVID-19 pandemic on physical activity and sedentary behavior in hypertensive older adults: An accelerometer-based analysis. Exp. Gerontol. 2020, 142, 111121. [CrossRef]

12. Panchal, N.; Kamal, R.; Cox, C.; Garfield, R. The Implications of COVID-19 for Mental Health and Substance Use. Kaiser Family Foundation (2020). Available online: https:/ / www.kff.org/coronavirus-covid-19/issue-brief/the-implications-of-covid-19-formental-health-and-substance-use/ (accessed on 20 April 2021).

13. Taquet, M.; Luciano, S.; Geddes, J.R.; Harrison, P.J. Bidirectional associations between COVID-19 and psychiatric disorder: Retrospective cohort studies of 62354 COVID-19 cases in the USA. Lancet Psychiatry 2021, 8, 130-140. [CrossRef]

14. Seven Ways to Prevent Falls in Memory Care during COVID-19 Pandemic. 2020. Available online: https://www.safely-you. com/wp-content/uploads/2020/04/QuickTips-COVID19.Final_.pdf (accessed on 15 December 2020).

15. Nasreddine, Z.S.; Phillips, N.A.; Bedirian, V.; Charbonneau, S.; Whitehead, V.; Collin, I.; Cummings, J.L.; Chertkow, H. The Montreal Cognitive Assessment, MoCA: A Brief Screening Tool For Mild Cognitive Impairment. J. Am. Geriatr. Soc. 2005, 53, 695-699. [CrossRef] [PubMed]

16. Weissman, M.M.; Sholomskas, D.; Pottenger, M.; Prusoff, B.A.; Locke, B.Z. Assessing depressive symptoms in five psychiatric populations: A validation study. Am. J. Epidemiol. 1977, 106, 203-214. [CrossRef] [PubMed]

17. Yardley, L.; Beyer, N.; Hauer, K.; Kempen, G.; Piot-Ziegler, C.; Todd, C. Development and initial validation of the Falls Efficacy Scale-International (FES-I). Age Ageing 2005, 34, 614-619. [CrossRef] [PubMed]

18. Powell Lawton, M.; Brody, E.M. Assessment of older people: Self-maintaining and instrumental activities of daily living. Gerontologist 1969, 9, 179-186. [CrossRef]

19. Beck, A.T.; Epstein, N.; Brown, G.; Steer, R.A. An inventory for measuring clinical anxiety: Psychometric properties. J. Consult. Clin. Psychol. 1988, 56, 893-897. [CrossRef]

20. Jørstad, E.C.; Hauer, K.; Becker, C.; on behalf of the ProFaNE Group; Msc, E.C.J.; Lamb, S.E. Measuring the Psychological Outcomes of Falling: A Systematic Review. J. Am. Geriatr. Soc. 2005, 53, 501-510. [CrossRef] 
21. Razjouyan, J.; Naik, A.D.; Horstman, M.J.; Kunik, M.E.; Amirmazaheri, M.; Zhou, H.; Sharafkhaneh, A.; Najafi, B. Wearable Sensors and the Assessment of Frailty among Vulnerable Older Adults: An Observational Cohort Study. Sensors 2018, 18, 1336. [CrossRef]

22. Najafi, B.; Crews, R.T.; Wrobel, J.S. Importance of Time Spent Standing for Those at Risk of Diabetic Foot Ulceration. Diabetes Care 2010, 33, 2448-2450. [CrossRef]

23. Najafi, B.; Armstrong, D.G.; Mohler, J. Novel Wearable Technology for Assessing Spontaneous Daily Physical Activity and Risk of Falling in Older Adults with Diabetes. J. Diabetes Sci. Technol. 2013, 1147-1160. [CrossRef]

24. Razjouyan, J.; Najafi, B.; Horstman, M.; Sharafkhaneh, A.; Amirmazaheri, M.; Zhou, H.; Kunik, M.E.; Naik, A. Toward Using Wearables to Remotely Monitor Cognitive Frailty in Community-Living Older Adults: An Observational Study. Sensors 2020, 20, 2218. [CrossRef]

25. Lindberg, C.M.; Srinivasan, K.; Gilligan, B.; Razjouyan, J.; Lee, H.; Najafi, B.; Canada, K.J.; Mehl, M.R.; Currim, F.; Ram, S.; et al. Effects of office workstation type on physical activity and stress. Occup. Environ. Med. 2018, 75, 689-695. [CrossRef]

26. Razjouyan, J.; Lee, H.; Parthasarathy, S.; Mohler, J.; Sharafkhaneh, A.; Najafi, B. Improving Sleep Quality Assessment Using Wearable Sensors by Including Information From Postural/Sleep Position Changes and Body Acceleration: A Comparison of Chest-Worn Sensors, Wrist Actigraphy, and Polysomnography. J. Clin. Sleep Med. 2017, 13, 1301-1310. [CrossRef] [PubMed]

27. Najafi, B.; Aminian, K.; Paraschiv-Ionescu, A.; Loew, F.; Büla, C.J.; Robert, P. Ambulatory system for human motion analysis using a kinematic sensor: Monitoring of daily physical activity in the elderly. IEEE Trans. Biomed. Eng. 2003, 50, 711-723. [CrossRef] [PubMed]

28. Parvaneh, S.; Mohler, J.; Toosizadeh, N.; Grewal, G.S.; Najafi, B. Postural Transitions during Activities of Daily Living Could Identify Frailty Status: Application of Wearable Technology to Identify Frailty during Unsupervised Condition. Gerontology 2017, 63, 479-487. [CrossRef]

29. Cohen, J. Statistical Power Analysis for the Behavioral Sciences; Academic Press: Cambridge, MA, USA, 2013.

30. Rosenthal, J.A. Qualitative Descriptors of Strength of Association and Effect Size. J. Soc. Serv. Res. 1996, 21, 37-59. [CrossRef]

31. Edwards, M.K.; Loprinzi, P.D. Effects of a Sedentary Behavior-Inducing Randomized Controlled Intervention on Depression and Mood Profile in Active Young Adults. Mayo Clin. Proc. 2016, 91, 984-998. [CrossRef] [PubMed]

32. Endrighi, R.; Steptoe, A.; Hamer, M. The effect of experimentally induced sedentariness on mood and psychobiological responses to mental stress. Br. J. Psychiatry 2016, 208, 245-251. [CrossRef]

33. De Bruin, E.D.; Najafi, B.; Murer, K.; Uebelhart, D.; Aminian, K. Quantification of everyday motor function in a geriatric population. J. Rehabil. Res. Dev. 2007, 44, 417-428. [CrossRef] 